\title{
LA-UR-15-23248
}

Approved for public release; distribution is unlimited.

Title: $\quad$ SESAME Equations of State for "Epoxy"

Author(s): $\quad$ Coe, Joshua Damon

Intended for: Report

Issued: 
Disclaimer:

Los Alamos National Laboratory, an affirmative action/equal opportunity employer,is operated by the Los Alamos National Security, LLC for the National NuclearSecurity Administration of the U.S. Department of Energy under contract DE-AC52-06NA25396. By approving this article, the publisher recognizes that the U.S. Government retains nonexclusive, royalty-free license to publish or reproduce the published form of this contribution, or to allow others to do so, for U.S. Government purposes. Los Alamos National Laboratory requests that the publisher identify this article as work performed under the auspices of the U.S. Departmentof Energy. Los Alamos National Laboratory strongly supports academic freedom and a researcher's right to publish; as an institution, however, the Laboratory does not endorse the viewpoint of a publication or guarantee its technical correctness. 


\section{SESAME Equations of State for "Epoxy"}

Joshua D. Coe ${ }^{a}$

Theoretical Division, Los Alamos National Laboratory, Los Alamos, NM, 87545

(Dated: 23 April 2015)

I examine LANL equations of state (EOS) for Epon 828, nominally with and without addition of Jeffamine T-403 curing agent. I review the methodology used to construct the existing EOS, as well as that of a new developmental SESAME table (97605) for the decomposition products of cured epoxy. I compare shock loci extracted from all tables with data, and comment on their relative merits.

a) Electronic mail: jcoe@lanl.gov 


\section{INTRODUCTION}

I will briefly review the status of LANL equations of state (EOS) for Epon 828 and its mixture with the curing agent Jeffamine T-403. I will also introduce a developmental EOS table for the decomposition products of the cured epoxy.

In Table I I list the numbers, authors, dates, and basic grid details of the relevant SESAME $^{1}$ tables. The density domain shrinks as one progresses through the series, and 7603 is noticeably more sparse than the previous two. The temperature domains are identical, but 7603 is the most dense by a wide margin.

The first issues to resolve (as is often the case with legacy "soft" materials) were those of composition and nomenclature. All three entries were dubbed "epoxy". Two of those cases (7601-2) were also described as Epon 828, a liquid resin derived from bisphenol A and epichlorohydrin, while the third (7603) was based on a 70/30 wt\% mixture of Epon 828 and Jeffamine T-403 curing agent. The normalized chemical composition, mean atomic weights $(\bar{A})$, mean atomic numbers $(\bar{Z})$, and reference densities $\left(\rho_{0}\right)$ read from the comment tables are given in Table II. The composition underlying 7603 was actually given by wt $\%$ in its 101 subtable, so I've translated this to mol\% in Table II. The $\bar{A}$ and $\bar{Z}$ I obtained agreed with those of the SESAME table to at least 3 significant digits, so I trust that my composition is essentially identical to that assumed previously. The compositions underlying 7601-2 and 7603 differ visibly from one another, but not dramatically so. Somewhat perplexing is the fact that 7601-2 are described simply as Epon 828, yet they contain nitrogen whereas pure Epon 828 does not. The initial densities differ by roughly 2.5\%. My suspicion is that all three tables were really meant to represent the cured material (i.e., that mixed with Jeffamine), but that this distinction was not appreciated when constructing the earlier tables.

\section{EQUATION OF STATE METHODOLOGY}

\section{A. Inert}

The only documentation for 7601-2 is found in their 102 subtables, but 7603 is thoroughly detailed in a 1994 tech report. ${ }^{2}$ All three are based on the SESAME decomposition of the Helmholtz free energy ${ }^{1,3,4}$

$$
F(\rho, T)=\phi_{0}+F_{\text {ion }}+F_{\text {elec }} .
$$


TABLE I. Summary of new (developmental) and existing SESAME tables for epoxy. The last column indicates whether the composition is based on neat Epon 828 or its $70 / 30$ wt\% mixture with Jeffamine T-403 curing agent.

\begin{tabular}{lccccc}
\hline \hline SESAME & Subtables & Grid $(\mathrm{n} \rho \times \mathrm{n} T)$ & Domain $(\mathrm{g} / \mathrm{cc}, \mathrm{K})$ & Author $($ Year $)$ & Cured? \\
\hline 7601 & $101-2,301-6$ & $69 \times 37$ & $\rho=0-2.37 \mathrm{E} 4$ & J. D. Johnson $(1984)$ & $?$ \\
& & & $T=0-1.16 \mathrm{E} 9$ & & \\
7602 & $101-2,301$ & $66 \times 27$ & $\rho=0-1.32 \mathrm{E} 3$ & J. D. Johnson $(1984)$ & $?$ \\
& & & & \\
7603 & $101-2,301-6,401$ & $37 \times 40$ & $\rho=0-1.16 \mathrm{E} 9$ & & \\
& & & $T=0-1.16 \mathrm{E} 9$ & & \\
97605 & 301 & $30 \times 29$ & $\rho=1.00975-3.2$ & present work & Y \\
& & $T=430-8125$ & & \\
\hline \hline
\end{tabular}

Here, $\phi_{0}$ is the $0 \mathrm{~K}$ energy of the static lattice, $F_{\text {ion }}$ is the contribution of the ions (including zero-point energy), and $F_{\text {elec }}$ stems from thermal excitation of the electrons. Coupling of $F_{\text {ion }}$ and $F_{\text {elec }}$ is neglected. Cold curves were obtained by fitting to shock data, that for the nominally uncured being taken from the LASL Compendium ${ }^{6}$ and the cured from Ref. 7. Electronic contributions were in both cases based on Thomas-Fermi-Dirac theory, ${ }^{8-10}$ using either united atom (7601-2) or additive volume (7603) methodologies. The earlier EOS used a generalized Chart D model ${ }^{11}$ for both Grüneisen $\Gamma$ and ionic contributions, whereas 7603 used generalized Chart D for Grüneisen and JDJNuc ${ }^{12}$ for the ionic model. In both cases, $\Gamma_{0}=0.8$. Although the 102 subtable for 7602 states that it is merely a recreation of 7601 with van der Waals loops removed, for reasons given below (Section. III) I don't think this is quite correct. See Ref. 2 for additional details on the construction of 7603.

TABLE II. Final compositions (per mol, normalized) and reference densities for the materials under survey.

\begin{tabular}{lcccc}
\hline \hline Material & Composition & $\overline{\mathrm{A}}$ & $\overline{\mathrm{Z}}$ & $\rho_{0}(\mathrm{~g} / \mathrm{cc})$ \\
\hline Epon 828 (uncured) & $\mathrm{C}_{0.347} \mathrm{H}_{0.577} \mathrm{~N}_{0.028} \mathrm{O}_{0.048}$ & 5.9134 & 3.2407 & 1.185 \\
Epon 828 (cured) & $\mathrm{C}_{0.376} \mathrm{H}_{0.534} \mathrm{~N}_{0.013} \mathrm{O}_{0.077}$ & 6.46450 & 3.49533 & 1.154 \\
\hline \hline
\end{tabular}




\section{B. Decomposition Products}

Epoxy decomposition products were modeled under the assumption of full chemical and thermodynamic equilibrium. In keeping with the equilibrium assumption and the location of relevant states on the carbon phase diagram, solid constituents were treated as diamond. In reality, of course, the situation is far more complicated.

The bulk free energy of fluid components was calculated with Ross perturbation theory ${ }^{13}$ based on an EXP6 potential. ${ }^{14}$ The latter has the form

$$
\phi(r)=\frac{\epsilon}{\alpha-6}\left[6 e^{-\alpha\left(\frac{r}{r_{0}}-1\right)}-\alpha\left(\frac{r_{0}}{r}\right)^{6}\right],
$$

where $\alpha$ sets the steepness of the repulsive wall, $\epsilon$ represents the energetic well depth, and $r_{0}$ gives the separation at which the intermolecular potential is minimized. EXP6 parameters for each species were calibrated to shock data on the pure fluid, ${ }^{15}$ and are listed in Table III. While I started with the full list shown there, I progressively pruned constituents whose mol number was everywhere two orders of magnitude down from the rest in order to facilitate numerical robustness. This yielded a fairly simple material comprising only the bold species in Table III. Fluids were combined in the form of an ideal mixture. ${ }^{16}$

The EOS for bulk diamond $\left(\rho_{0}=3.51 \mathrm{~g} / \mathrm{cm}^{3}\right)$ was of Mie-Grüneisen form ${ }^{17}(\Gamma=0.88)^{18}$ built from a Murnaghan ${ }^{19}$ cold curve $\left(B_{0}=440 \mathrm{GPa}, N=3.65\right)^{20}$ and a Debye thermal ionic contribution ${ }^{21}\left(\theta_{D}=1850 \mathrm{~K}\right)^{18}$; thermal electronic contributions to the free energy are negligible over the relevant domain and therefore were neglected. In keeping with fairly standard practice, ${ }^{22} \Gamma / V$ was assumed constant. The free energy of the complete mixture (fluids+solid) was minimized as a function of chemical composition subject to conservation and non-negativity of elemental mass fractions. I have no real data on epoxy heat of formation, so it was simply set to zero.

\section{HUGONIOTS}

In Figures 1-2, I compare Hugoniots interpolated from the relevant SESAME tables with shock data ${ }^{6,7}$ in the $U_{s}-u_{p}$ and $P-V$ planes. In all plots I have added considerably to the

grid for the sake of obtaining smooth curves. Ambient sound speeds read from 7601-3 are roughly $20 \%, 5 \%$, and $2 \%$ (respectively) higher than the experimental value of $2.265 \mathrm{~km} / \mathrm{s}^{6}$ 
TABLE III. EXP6 parameters (see eqn. 2) of fluids comprising the decomposition products mixture for epoxy. ${ }^{15}$ All constituents were included initially, but only those in bold were retained for construction of the final table. Pair potentials were translated to bulk free energies using thermodynamic perturbation theory. ${ }^{13}$

\begin{tabular}{lccc}
\hline \hline Component & $r_{0} / \AA$ & $\left(\epsilon / \mathrm{k}_{B}\right) / \mathrm{K}$ & $\alpha$ \\
\hline $\mathrm{N}_{2}$ & 4.0876 & 128.24 & 12.936 \\
$\mathrm{NO}$ & 3.995 & 117.1 & 12.08 \\
$\mathrm{O}_{2}$ & 4.11 & 75.0 & 13.117 \\
$\mathrm{CO}_{2}$ & 4.096 & 335.0 & 13.781 \\
$\mathbf{H}_{2} \mathbf{O}$ & 3.5 & 424.0 & 10.0 \\
$\mathbf{C O}$ & 4.0876 & 128.24 & 12.936 \\
$\mathrm{CH}_{4}$ & 4.22 & 154.1 & 12.5 \\
$\mathbf{H}_{2}$ & 3.43 & 36.4 & 11.1 \\
$\mathrm{HCO}_{2} \mathrm{H}$ & 4.096 & 335.0 & 13.781 \\
$\mathrm{NH}_{3}$ & 3.3 & 424.0 & 12.0 \\
$\mathrm{~N}_{2} \mathrm{O}$ & 4.096 & 335.0 & 13.781 \\
$\mathrm{C}_{2} \mathrm{~N}_{2}$ & 4.9 & 330.0 & 13.0 \\
\hline \hline
\end{tabular}

Concavity at low particle velocity is a common Hugoniot feature of soft materials such as epoxy. ${ }^{17}$. All four tables do a reasonable job with the data at high particle velocity.

Both data sets display a discontinuity begining around $u_{p} \approx 3 \mathrm{~km} / \mathrm{s}$. While 7602 transitions smoothly from reactant to products, 7601 and 7603 preserve the cusp. They achieve this, however, through adjustment of the cold curve (which was explicitly constructed to recover the Hugoniot, see Section II A), as shown in Figure 3. This highlights one of the pathologies of using a single table to describe both an organic solid and its decomposition products: the transition from one to the other is completely independent of temperature, and persists even at $0 \mathrm{~K}$. SESAME 7602 lacks a 306 subtable, but its smoothness over the corresponding stretch of particle velocities belies its being merely a "Maxwellized" version of 7601 . 


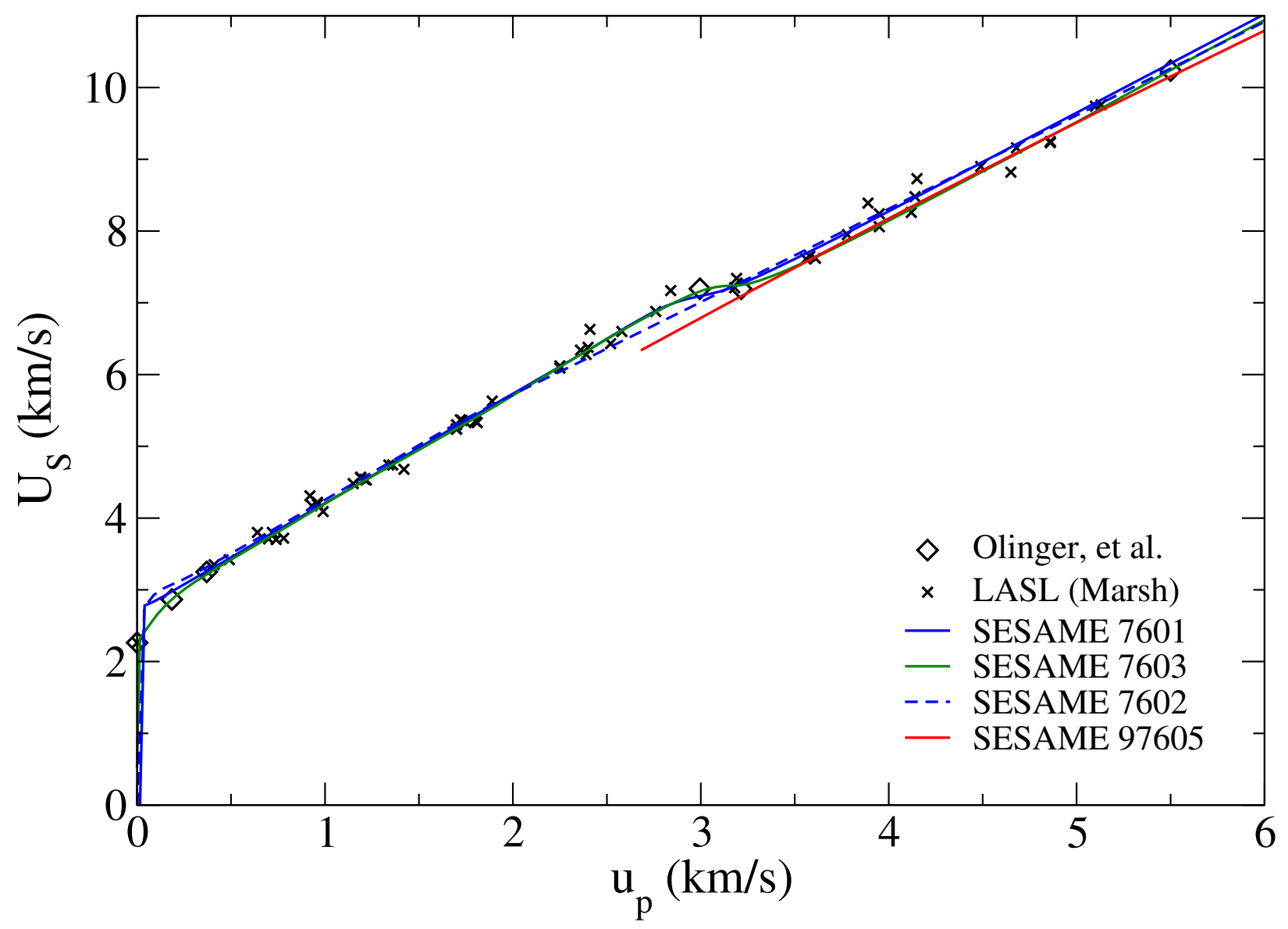

FIG. 1. Hugoniots in the $U_{s}-u_{p}$ plane, as read from SESAME tables for epoxy. Results are compared with shock data taken from Refs. 6 and 7 . 


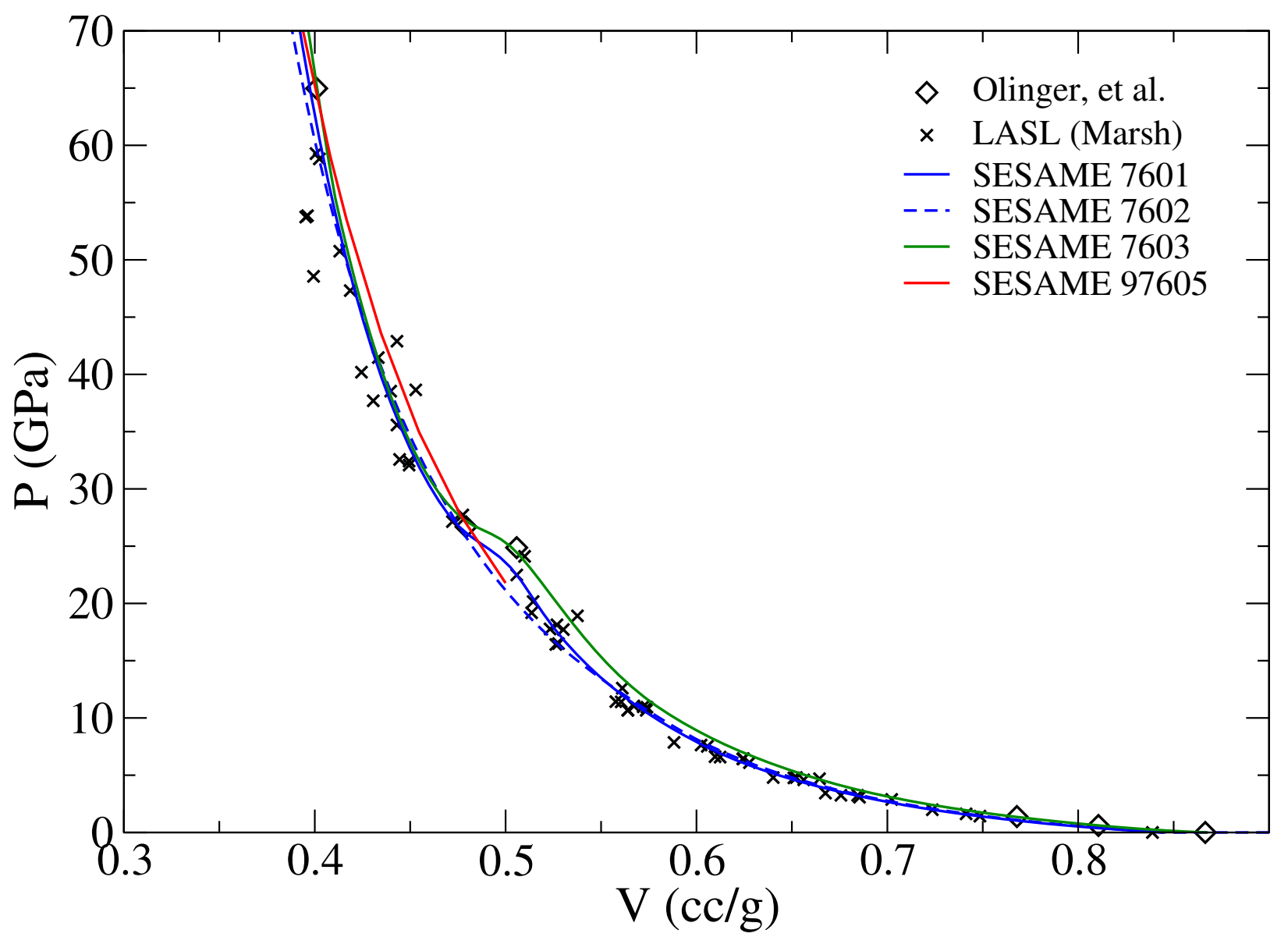

FIG. 2. Hugoniots in the $P-V$ plane, as read from SESAME tables for epoxy. Results are compared with shock data taken from Refs. 6 and 7. 


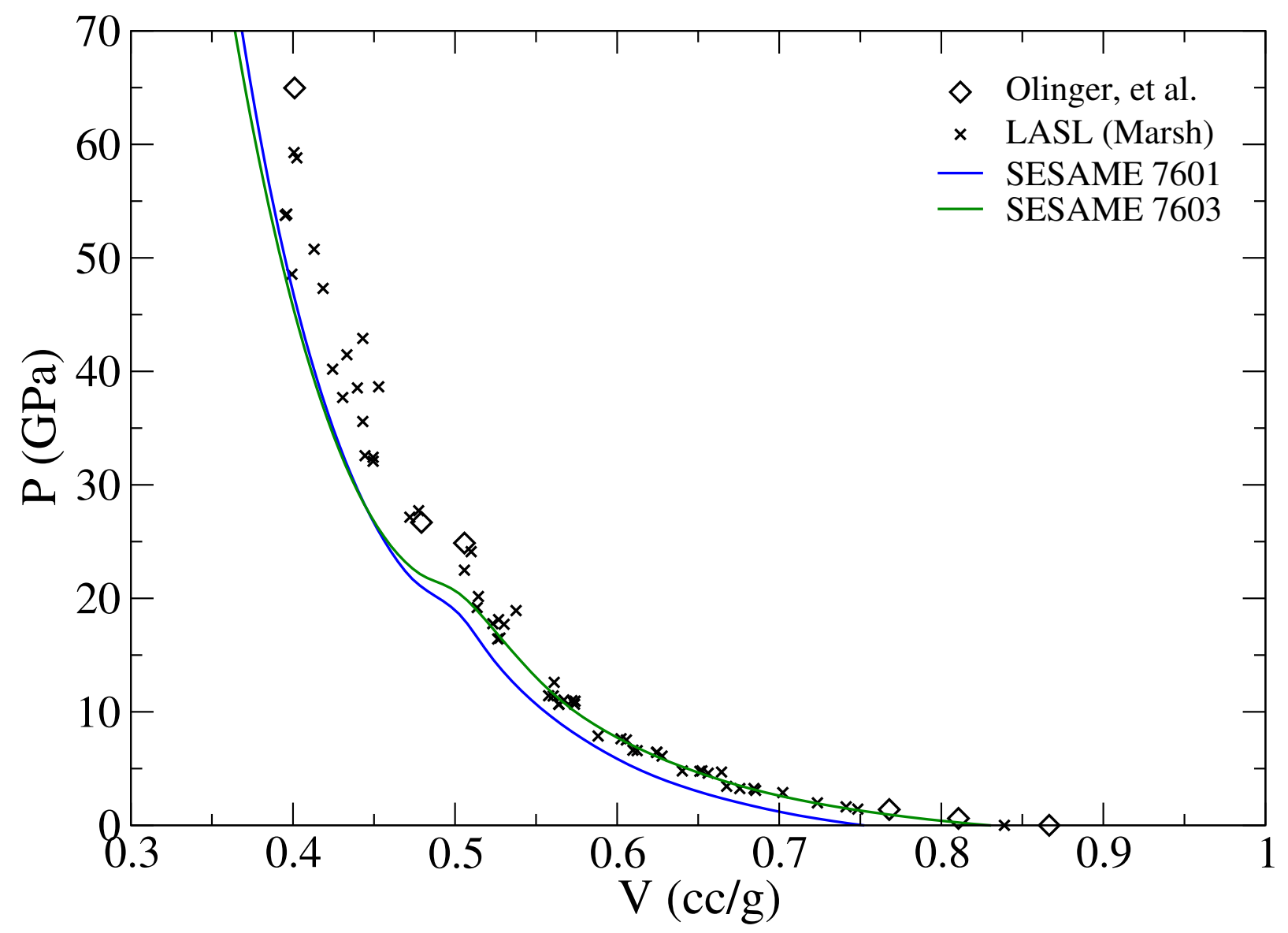

FIG. 3. Cold curves read from SESEAME 7601 and 7603, as compared with shock data taken from Refs. 6 and 7. 


\section{SUMMARY}

I have generated a developmental SESAME table (97605) for epoxy decomposition products, and reviewed the origin and quality of epoxy tables (7601-3) already in the official repository. Here are some conclusions:

- The experimental basis for all these tables is extremely thin, and appears to consist entirely of shock and sound speed data.

- Of the existing tables 7601-3, only 7603 is based on the correct chemical composition. Proper adjustment would most likely constitute a small correction to 7601-2. The composition underlying 97605 is the same as that used to construct 7603 .

- Of the existing tables 7601-3, 7603 does the best job of reproducing the ambient sound speed.

- Of the existing tables 7601-3, only 7602-3 attempt to recover the Hugoniot discontinuity believed due to decomposition. However, their means of doing so is physically unjustifiable.

\section{ACKNOWLEDGEMENTS}

I would like to thank Tinka Gammel (T-1) for some very helpful technical assistance.

\section{REFERENCES}

${ }^{1}$ S. P. Lyon and J. D. Johnson, "SESAME: The Los Alamos National Laboratory Equation of State Database," LA-UR-92-3407 (Los Alamos National Laboratory, 1992).

${ }^{2}$ J. C. Boettger, "Sesame Equation of State for Epoxy," LA-12755-MS (Los Alamos National Laboratory, 1994).

${ }^{3}$ J. Abdallah, "User's Manual for Grizzly," LA-10244-M (Los Alamos National Laboratory, 1984).

${ }^{4}$ G. I. Kerley, "User's Manual for Panda: A Computer Code for Calculating Equations of State," LA-8833-M (Los Alamos National Laboratory, 1981). 
${ }^{5}$ B. I. Bennett, "On Obtaining the Zero-Temperature Equation of State from Shock Data," LA-9890-MS (Los Alamos National Laboratory, 1984).

${ }^{6}$ S. P. Marsh, ed., LASL Hugoniot Data (University of California Press, 1980).

${ }^{7}$ B. Olinger, J. Fritz, and C. E. Morris, "Equations of State for PEEK, Epon 828, and Carbon Fiber-Epon Composite," (1993), Los Alamos National Laboratory. As cited in Ref. 2.

${ }^{8}$ R. P. Feynman, N. Metropolis, and E. Teller, Phys. Rev. 75 (1949).

${ }^{9}$ R. D. Cowan and J. Ashkin, Phys. Rev. 105 (1957).

${ }^{10}$ Ref. 8 describes the basic algorithm for Thomas-Fermi, and Ref. 9 describes the exchange contribution. However, what is actually implemented in Grizzly is described Appendix A of Ref. 3, which differs from Ref. 9 in its use of a zero-temperature expression for exchange at all temperatures.

${ }^{11}$ S. L. Thompson and H. S. Lawon, "Improvements to the Chart-D Radiational Hydrodynamics Code III: Revised Analytic Equations of State," SC-RR-710714 (Sandia National Laboratory, 1972).

${ }^{12}$ J. D. Johnson, High Pressure Research 6, 277 (1991).

${ }^{13}$ M. Ross, J. Chem. Phys. 71, 1567 (1979).

${ }^{14}$ A. J. Stone, The Theory of Intermolecular Forces (Oxford University Press, 1996) See section 11.1.3.

${ }^{15}$ M. S. Shaw, Los Alamos National Laboratory, unpublished.

${ }^{16}$ J. S. Rowlinson and F. L. Swinton, Liquids and Liquid Mixtures, 3rd ed. (Butterworth Scientific, 1982) . A macroscopic treatment of ideal mixtures is given in 4.3, a microscopic one in 8.2 .

${ }^{17}$ R. Menikoff, in Shock Wave Science and Technology Reference Library, edited by Y. Horie (Springer, 2007) Chap. 4.

${ }^{18}$ J. D. Coe, Los Alamos National Laboratory, unpublished.

${ }^{19}$ F. D. Murnaghan, Proc. Natl. Acad. Sci. USA 30, 244 (1944).

${ }^{20}$ A. Dewaele, F. Datchi, P. Loubeyre, and M. Mezouar, Phys. Rev. B 77, 094106 (2008).

${ }^{21}$ D. A. Mcquarrie, Statistical Mechanics (University Science Books, 1976) . See Sec. 11-3.

${ }^{22}$ R. G. McQueen, S. P. Marsh, J. W. Taylor, J. N. Fritz, and W. J. Carter, in High Velocity Impact Phenomena, edited by R. Kinslow (Academic Press, 1970) Chap. VII, pp. 293-417. 
${ }^{23}$ S. Crockett, "Extending High Explosive Equations of State," LA-UR-06-5738 (Los Alamos National Laboratory, 2006). 